\title{
Analysis, Design, and Optimisation of an Electromagnetic Energy Harvester
}

\author{
Uchenna DIALA, Yiling LU and Amar BOUSBAINE \\ University of Derby, Markeaton Street, DE223AW, UK
}

\begin{abstract}
One of two major limitations of a vibration energy harvester (VEH), concerns its limited performance due to its confined physical enclosure. The maximum span realizable is attained at a specific excitation level. This excitation level provides the maximum energy harvested by the VEH device. Due to span constraints, VEHs are designed to operate at the maximum span achievable at the maximum excitation level existing within the region of interest. In this study, a constrained optimisation problem (for the VEH) is formulated and investigated. This paper focuses on the analysis, design and optimisation of a nonlinear VEH device.
\end{abstract}

Keywords: Vibration, Vibration energy harvester, nonlinear damping, OFRF.

\section{Introduction}

Vibration energy harvesting has recently received substantial consideration due to the rapid developments in technologies such as low-power wireless sensors [1, 2]. Two major limitations exist for most of the VEHs. The first and more considered limitation is the operational bandwidth of a VEH. VEHs are typically designed to operate at their natural frequencies. This implies excitation frequencies beyond the natural frequency reduces the efficiency of the harvester [3-5]. The second limitation concerns the constrained physical enclosure of the $\mathrm{VEH}$, which causes the suspended mass to oscillate within a specified span $[6,7]$. Therefore, for best performance, the maximum excitation level is considered at the design stage. However, excitations below the maximum value reduces the energy harvested. A recent study in [8] revealed that a nonlinear cubic electrical damping extends the harvestable power of a VEH. It was shown that by integrating a cubic nonlinear damper to a VEH system, it outperformed an equivalent linear VEH. The results obtained in [8] indicated that at maximum excitation, the same relative displacement of the $\mathrm{VEH}$ and hence average power, are provided by both nonlinear and equivalent linear VEHs. Nevertheless, at excitations below the maximum level, the nonlinear VEH provided more energy compared to its linear equivalent. The study in [9] corroborated this assertion and demonstrated the effect of the harvester coil resistance on its harvestable power level.

This study focuses on the analysis, design, and optimisation of a VEH integrated with a cubic damping nonlinearity. A mathematical model of the nonlinear VEH is developed and analysed, using the Output Frequency Response Function (OFRF) method. Performance metrics of the VEH, such as average power, are expressed in terms of the parameter of interest, using the OFRF method. Subsequently, an optimisation method is conducted to maximize the energy harvested by the VEH, subject to span constraint. 


\section{Model formulation}

In this study, a base-excited single degree-of-freedom (SDOF) VEH is considered, as illustrated in Figure 1. The SDOF system is seen to have an oscillating mass, $m$, basedisplacement $y(t)$, spring stiffness $k_{1}$, the relative displacement between the oscillating mass and the support-base of the harvester $z(t)=x(t)-y(t)$, equivalent linear viscous damping coefficient $c_{1}$ and nonlinear cubic damping coefficient $c_{3}$.

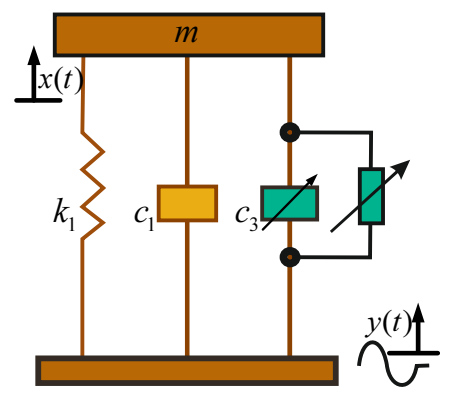

Figure 1. SDOF base-excited VEH with a nonlinear damper.

The dynamic equation [8] is given as

$$
m \ddot{z}+c_{1} \dot{z}+c_{3} \dot{z}^{3}+k_{1} z=-m \ddot{y}
$$

Supposing a harmonic base excitation, $y(t)=Y \sin (\omega t)$ is assumed with magnitude, $Y$, frequency, $\omega$ and zero phase shift. Then the dynamic equation of the VEH can be expressed as

$$
m \ddot{z}+c_{1} \dot{z}+c_{3} \dot{z}^{3}+k_{1} z=m \omega^{2} Y \sin (\omega t)
$$

The average power of the damping system is given as in [8]

$$
P_{a v}=\frac{1}{T} \int_{0}^{T}\left(c_{1} \dot{z}\right) \cdot \dot{z} d t+\frac{1}{T} \int_{0}^{T}\left(c_{3} \dot{z}^{3}\right) \cdot \dot{z} d t
$$

Considering a general mono-frequency oscillation, Eq. (3) becomes

$$
P_{a v}=\frac{1}{2} c_{1} \omega^{2} Z^{2}+\frac{3}{8} c_{3} \omega^{4} Z^{4}
$$

The first term of Eq. (4) is regarded as a loss based on the dissipative power of parasitic damping [10]. For such a loss, $c_{1}$ is the equivalent viscous damping coefficient for the parasitic damping [11]. The second term provides the harvestable power. Furthermore, in practical VEHs, the relative displacement of the oscillating mass is constrained by the physical enclosure of the system. This, implies that the magnitude of the relative displacement is constrained to a certain maximum value, $Z_{\max }$. This physical constraint however limits the maximum harvestable power as it is seen in Eq. (4) that the power harvested is a function of $Z$, and also, $Z$ is a function of the excitation frequency, $\omega$ [12]. Therefore, it is important that the damping parameter, $c_{3}$, to be designed, should be able to restrain the oscillations of the suspended mass within a tolerable span while maximising the energy harvested. The second term in Eq. (4) is given as 


$$
P_{a v v_{c_{3}}}=\frac{3}{8} c_{3} \omega^{4} Z^{4}
$$

The OFRF-based approach will be utilised in this work, for the analysis of the dynamic model of the VEH in Eq. (2). The parameter of interest, $c_{3}$, will also be designed and optimised to ensure the realization of the maximum harvestable power, subject to existing constraints. The benefit of the OFRF is that the method can provide an explicit analytical relationship between the design objective, $P_{a v c_{3}}$ and system parameter of interest, $c_{3}$. This can significantly simplify the system design and optimisation. The performance metrics sought in this work are the relative displacement, $Z$, and harvestable power, $P_{a v c_{3}}$. For an elaborate procedure on how an OFRF model is derived, refer to the following studies [13-17]. This has been omitted here due to page restrictions.

\section{System Analysis, Design and Optimisation}

The subsequent analysis and simulations have been performed using the following model parameters, $m=1 \mathrm{~kg}, c_{1}=0.035 \mathrm{~N} . \mathrm{s} . \mathrm{m}^{-1}, k_{1}=4 \pi^{2} \mathrm{~N} . \mathrm{m}^{-1}, Y_{\max }=0.4 \mathrm{~m}$ [8]. It is observed that Eq. (2) belongs to the class of the Volterra system as shown in eq. (8) of [15], with $M=3$ and $L=2$. The system parameters are identified as $c_{1,0}(2)=m, c_{1,0}(1)=c_{1}$, $c_{3,0}(111)=c_{3}, c_{1,0}(0)=k$, and $c_{0,1}(0)=-m \omega^{2} Y$, while other parameters are zero.

Therefore, the OFRF of Eq. (2) is derived, using the OFRF method, as

$$
Z(\mathrm{j} \omega)=\theta_{0}(\mathrm{j} \omega)+\theta_{1}(\mathrm{j} \omega) c_{3}+\theta_{1}(\mathrm{j} \omega) c_{3}^{2}+\cdots+\theta_{10}(\mathrm{j} \omega) c_{3}^{10}+\theta_{11}(\mathrm{j} \omega) c_{3}^{11}
$$

The coefficients $\theta_{0}, \theta_{1} \cdots \theta_{11}$ in Eq. (6) are complex values. However, these values have been omitted here due to page restrictions. The output frequency response, $Z$ is seen to be a function of $\omega$ and $c_{3}$, therefore can be written as $Z\left(\mathrm{j} \omega ; c_{3}\right)$. Note that the objective is to design $c_{3}$ that provides the maximum power harvested by the $\mathrm{VEH}$, considering existing span constraint. It is observed in Eq. (5) that the average power harvested is also a function of the output response, $Z$. Substituting Eq. (6) into Eq. (5), the OFRF of the average harvestable power becomes

$$
P_{a v \mid c_{3}}=\frac{3}{8} c_{3} \omega^{4}\left|Z\left(\mathrm{j} \omega ; c_{3}\right)\right|^{4}
$$

The OFRF is a polynomial representation of the system output. A comparison between the results obtained from the OFRF method and Runge-Kutta 4 algorithm (ODE45 in MATLAB), over the parameter value outside the OFRF training set (in this case, $c_{3}=0.0101 \mathrm{~N} . \mathrm{s}^{3} \cdot \mathrm{m}^{-3}$ ), is shown in Figure 2. The results indicate that the OFRF provides a very good representation of the actual system model. 

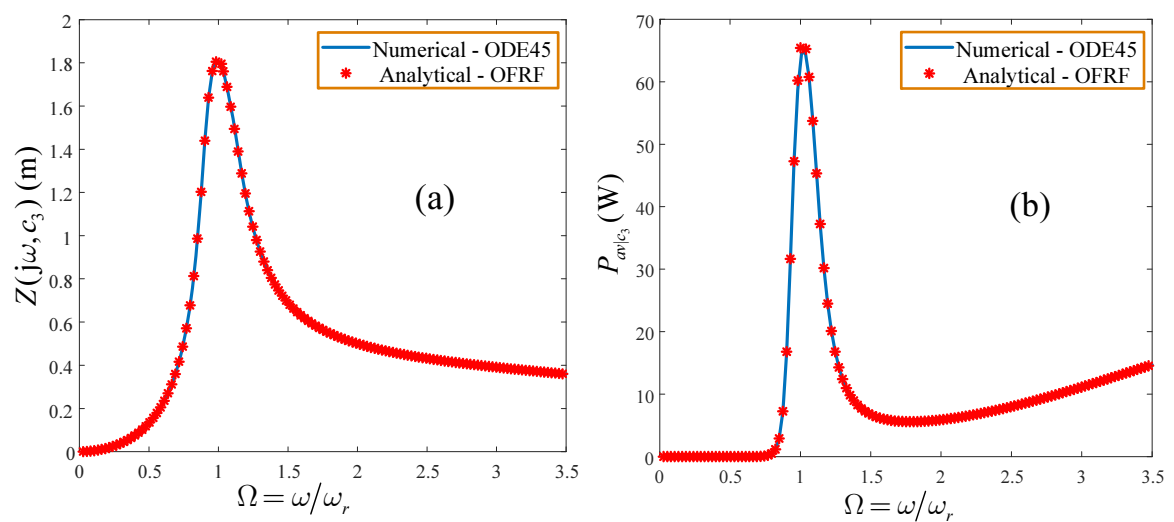

Figure 2. Comparison between the OFRF analytical and Runge-Kutta numerical solution: a) relative mass displacement, b) Average power harvested.

\subsection{Optimisation of an unconstrained VEH system}

It should be noted that in the absence of a mass displacement constraint in the VEH, the optimal value of $c_{3}$ required to maximise the energy harvested, $P_{a v c_{3}}$, can be obtained by evaluating the zero of the derivative of Eq. (7) with respect to $c_{3}$. This gives

$$
\frac{d P_{a v c_{3}}}{d c_{3}}=0
$$

Though there are several solutions to Eq. (8), the minimum, non-negative real solution is selected which is evaluated as $c_{3}^{\text {opt1 }}=0.0015 \mathrm{~N} \cdot \mathrm{s}^{3} \cdot \mathrm{m}^{-3}$. This value is substituted into Eqs. (6) and (7) to obtain the corresponding mass displacement, $\left|Z\left(\mathrm{j} \omega_{r} ; c_{3}\right)\right|=3.035 \mathrm{~m}$ and average harvestable power, $P_{a v c_{3} \max 1}=76.68 \mathrm{~W}$ of the $\mathrm{VEH}$, considering no constraints. However, practical VEHs are constrained in their relative displacement, $Z$ hence posing a constrained optimisation problem.

\subsection{Optimisation of a constrained VEH system}

From the derived OFRF representations for the average harvestable power and the output frequency response, the optimisation problem for the nonlinear parameter, $c_{3}$, subject to a constraint, can be formulated as;

$$
\begin{array}{ll}
\max _{c_{3}} & P_{a v c_{3}}\left(\omega_{r}, c_{3}\right) \\
\text { s.t. }\left\{\begin{array}{l}
\left|Z\left(\mathrm{j} \omega_{r} ; c_{3}\right)\right|-Z_{\max } \leq 0 \\
c_{3}-1 \times 10^{-2} \leq 0 \quad \text { where } \omega_{r}=6.3 \mathrm{rad} . \mathrm{s}^{-1}, Z_{\max }=2.5 \mathrm{~m}, \text { and } c_{3} \in[0,0.01]
\end{array}\right.
\end{array}
$$

Eq. (9) is solved using the MATLAB fmincon function. The optimal cubic damping is obtained as $c_{3}^{\text {opt2 }}=0.0032 \mathrm{~N} . \mathrm{s}^{3} \cdot \mathrm{m}^{-3}$. Substituting this value into Eqs. (6) and (7) yields $\left|Z\left(\mathrm{j} \omega_{r}\right)\right|=2.500 \mathrm{~m}$ and $P_{a v}=75.07 \mathrm{~W}$. To demonstrate the effectiveness of the OFRF method, the optimal damping solution, $c_{3}^{\text {opt2 }}$ is substituted into Eq. (2) and Eq. (5), which 
yields an output spectrum and corresponding average power of $\left|Z\left(\mathrm{j} \omega_{r}\right)\right|=2.501 \mathrm{~m}$ and $P_{a v}=74.98 \mathrm{~W}$, respectively. Table 1 shows the maximum average power harvestable by the VEH, while considering both unconstrained and constrained conditions.

Table 1. Maximum average harvestable power attainable at $\omega=6.3 \mathrm{rad} \mathrm{s}^{-1}(\Omega=1)$ subject to the system constraint, $Z_{\max }=2.5 \mathrm{~m}$

\begin{tabular}{cccc}
\hline $\begin{array}{c}\text { Constraint } \\
\text { present? }\end{array}$ & $\begin{array}{c}\text { Mass displacement of } \\
\text { VEH system, } Z(\mathbf{m})\end{array}$ & $\begin{array}{c}\text { Max. average harvestable } \\
\text { Power, } P_{a v c_{3} \max }(\mathbf{W})\end{array}$ & $\begin{array}{c}\text { Nonlinear damping, } \\
c_{3}^{\mathrm{opt}}\left(\mathrm{Ns}^{3} \mathrm{~m}^{-3}\right) \times 10^{-3}\end{array}$ \\
\hline No & 3.035 & 76.68 & 1.5 \\
Yes & 2.500 & 75.07 & 3.2 \\
\hline \hline
\end{tabular}

It is observed that the maximum average harvestable power of the VEH, considering the displacement constraint of the VEH, $Z=2.500 \mathrm{~m}$, is determined as $P_{a v c_{3} \max 2}=75.07 \mathrm{~W}$ for $c_{3}^{\text {opt2 }}=0.0032 \mathrm{~N} \cdot \mathrm{s}^{3} \cdot \mathrm{m}^{-3}$. Figure 3 shows the effect of a nonlinear cubic damping characteristic on, (a) the relative displacement, $Z$ and (b) average power, $P_{a v k_{3}}$ of the VEH, respectively. These graphs were obtained based on the variation of nonlinear cubic damping, $c_{3} \in[0,0.01] \mathrm{Ns}^{3} \mathrm{~m}^{-3}$, at the resonant frequency. In Figure $3 \mathrm{a}$, it is observed that $Z$ decreases monotonically as $c_{3}$ increases. There exists an inverse relationship between both parameters. That is, $Z$ decreases as $c_{3}$ increases and vice versa. However, in Eq. (5), it is observed that the average harvestable power is a function of both parameters, $Z$ and $c_{3}$. This implies that $P_{a v c_{3}}$ becomes zero if either of these parameters is zero. Therefore, as revealed in Figure 3b, an optimal value of the nonlinear damping parameter provides a maximum average harvestable power, subject to span constraint.

Figure 4 represents the system output response (a) and average harvestable power (b) obtained, for unconstrained and constrained cases. Figure 5 shows a surface plot variation of the average harvestable power with respect to excitation frequencies, and nonlinear damping characteristics.
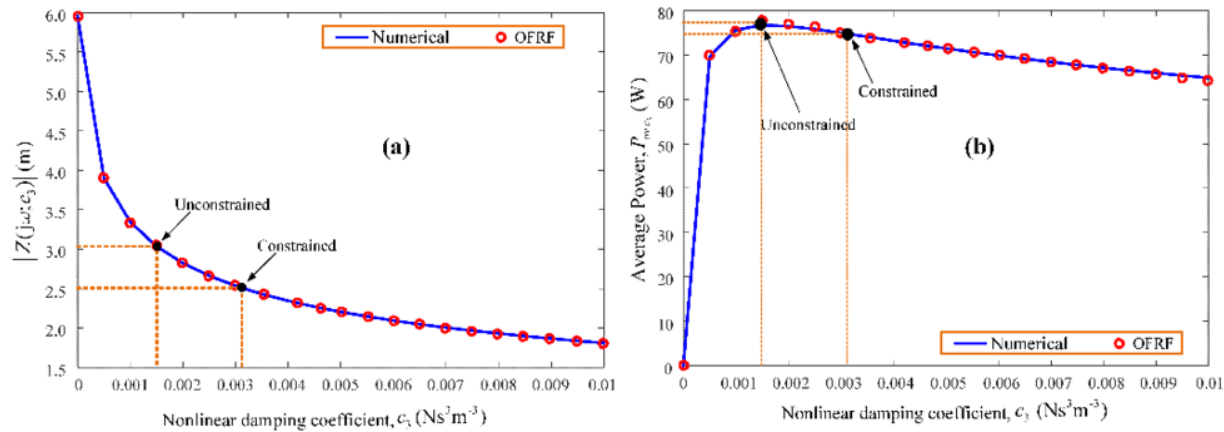

Figure 3. Comparison of analytical method (OFRF) and numerical simulations for (a) the output frequency response of Eq. (2), and (b) the average power of the $\mathrm{VEH}$. 

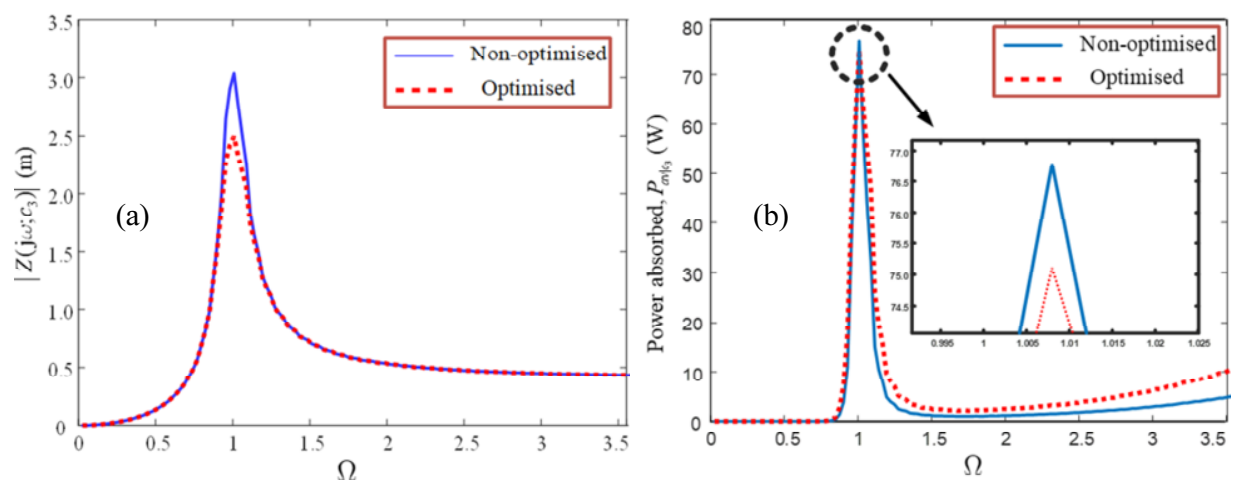

Figure 4. Comparison of (a) system output response and (b) average harvestable power obtained, for nonoptimised and optimised cases.
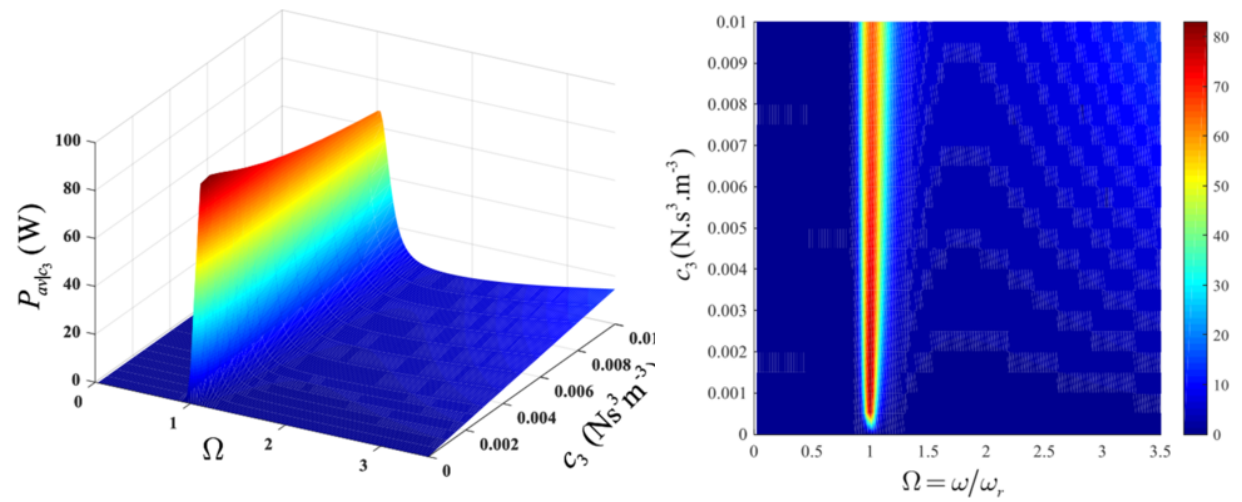

Figure 5. Average harvestable power obtained at different excitation frequencies, and nonlinear damping parameter values (b) Contour plot for average harvestable power

\section{Conclusions}

This work focused on the analysis, design and optimisation of a nonlinear VEH. The proposed VEH system was integrated with a nonlinear damping characteristic and its energy harvesting performance was investigated considering unconstrained and constrained conditions. It was observed that under unconstrained conditions, more power was harvested for a lower nonlinear damping level compared to the case under constrained conditions. Future studies will consider the extension of a VEH harvestable power under constrained conditions.

\section{References}

[1] Beeby S.P., Tudor M.J., and White N.M., "Energy harvesting vibration sources for microsystems applications". Measurement science and technology 17, no. 12 (2006): R175.

[2] Priya S. and Inman D.J., eds. Energy harvesting technologies. Vol. 21. New York: Springer, 2009. 
[3] Stephen N.G., "On energy harvesting from ambient vibration". Journal of sound and vibration 293, no. 1-2 (2006): 409-425.

[4] Beeby S.P., Torah R.N., Tudor M.J., Glynne-Jones P., O'donnell T., Saha C.R., and Roy S., "A micro electromagnetic generator for vibration energy harvesting". Journal of Micromechanics and Microengineering 17, no. 7 (2007): 1257.

[5] Diala U., Mofidian SM. M., Lang Z.Q., and Bardaweel H., "Analysis and optimal design of a vibration isolation system combined with electromagnetic energy harvester." Journal of Intelligent Material Systems and Structures 30, no. 16 (2019): 2382-2395.

[6] Hendijanizadeh M., Sharkh S.M., Elliott S.J., and Moshrefi-Torbati M., "Output power and efficiency of electromagnetic energy harvesting systems with constrained range of motion". Smart Materials and Structures 22, no. 12 (2013): 125009 .

[7] Hendijanizadeh M., Moshrefi-Torbati M., and Sharkh S.M., "Constrained design optimization of vibration energy harvesting devices". Journal of Vibration and Acoustics 136, no. 2 (2014): 021001.

[8] Tehrani M.G. and Elliott S.J., "Extending the dynamic range of an energy harvester using nonlinear damping". Journal of Sound and Vibration 333, no. 3 (2014): 623-629.

[9] Hendijanizadeh M., Elliott S.J., and Ghandchi-Tehrani M., "The effect of internal resistance on an energy harvester with cubic resistance load". The 22nd International Congress on Sound and Vibration (ICSV22), Italy, (2015).

[10] Simeone L., Ghandchi-Tehrani M., Elliott S.J., and Hendijanizadeh M., "Nonlinear damping in an energy harvesting device". Conference on Noise and Vibration Engineering (ISMA2014), Belgium, (2014).

[11] Zhu S., Shen W.A., and Xu Y.L., "Linear electromagnetic devices for vibration damping and energy harvesting: Modeling and testing". Engineering Structures 34 (2012): 198-212.

[12] Williams C.B., and Yates R.B., "Analysis of a micro-electric generator for microsystems". Sensors and Actuators A: Physical 52, no. 1-3 (1996): 8-11.

[13] Peng Z.K. and Lang Z.Q., "The effects of nonlinearity on the output frequency response of a passive engine mount". Journal of Sound and Vibration 318, no. 1-2 (2008): 313-328.

[14] Zhu Y. and Lang Z.Q., "Design of Nonlinear Systems in the Frequency Domain: An Output Frequency Response Function-Based Approach". IEEE Transactions on Control Systems Technology 26, no. 4 (2018): 1358-1371.

[15] Lang Z.Q., Billings S.A., Yue R., and Li J., "Output frequency response function of nonlinear Volterra systems". Automatica 43, no. 5 (2007): 805-816.

[16] Jing X.J., Lang Z.Q., and Billings S.A., "Output frequency response function-based analysis for nonlinear Volterra systems". Mechanical Systems and Signal Processing 22, no. 1 (2008): 102-120.

[17] Jing X.J., Lang Z.Q., Billings S.A., and Tomlinson G.R., "The parametric characteristic of frequency response functions for nonlinear systems". International Journal of Control 79, no. 12 (2006): 1552-1564. 